Beyond Philology No. 17/4, 2020

ISSN 1732-1220, eISSN 2451-1498

https://doi.org/10.26881/bp.2020.4.01

\title{
Translating a pandemic: \\ A corpus study of COVID-19 multi-word terminology in EU press releases
}

\author{
ALEKSANDRA TOMASZEWSKA \\ NATALIA ZAWADZKA-PALUEKTAU
}

Received 7.11.2020, received in revised form 1.12.2020, accepted 1.12.2020.

\begin{abstract}
The study employs a parallel English-Polish corpus to investigate how COVID-19 multi-word terms are handled in translations of EU press releases. Translation techniques are examined at four levels of analysis: (1) term variation, (2) institutionalization, (3) domestication/foreignization, and (4) degree of transfer of information. The results are discussed in regard to the characteristics of COVID-19 terminology and its, often neological, instability, which manifests itself in high levels of terminological variation, inconsistent use of recommended institutional equivalents, and varied degrees of information transfer between SL and TL. The findings are also reviewed in light of the nature of press releases which, as an essential link in the transmission of knowledge from EU institutions to citizens, prompt the use of domesticating techniques.
\end{abstract}




\title{
Keywords
}

COVID-19, terminology, translation techniques, EU translation, institutionalization, terminological variation

\section{Pandemia w tłumaczeniu: analiza korpusowa terminologii wielowyrazowej dotyczącej COVID-19 w komunikatach prasowych UE}

\begin{abstract}
Abstrakt
W niniejszym artykule wykorzystano równoległy korpus angielsko-polski do zbadania, jak w tłumaczeniach komunikatów prasowych UE oddawane sa terminy złożone zwiazane $z$ COVID-19. Techniki tłumaczeniowe zbadano na czterech poziomach analizy: (1) zróżnicowania terminologicznego, (2) instytucjonalizacji, (3) udomowienia/egzotyzacji oraz (4) stopnia przekazywania informacji. W badaniu uwzględniono charakterystykę terminologii dotyczącej COVID-19, w tym jej niestabilność (często neologiczna), przejawiającą się dużym zróżnicowaniem terminologicznym, niekonsekwentnym stosowaniem zalecanych odpowiedników instytucjonalnych oraz przesunięciami w przekazie informacji z języka źródłowego do docelowego. Jednocześnie wyniki interpretowano w odniesieniu do specyfiki komunikatów prasowych, które, pełniac rolę istotnego ogniwa w przekazywaniu wiedzy $z$ instytucji UE do obywateli, skłaniaja do stosowania technik udomawiajacych.
\end{abstract}

\section{Słowa kluczowe}

COVID-19, terminologia, techniki tłumaczeniowe, tłumaczenie UE, instytucjonalizacja, zróżnicowanie terminologiczne

\section{Introduction}

Terminology provides essential access points to specialized knowledge structures (Faber 2009: 109). As such, it contributes significantly to the meaning-making of texts (Biel and Koźbiał 
2020). During the COVID-19 health crisis, terms have played a significant role in conveying information from experts to the general public. This has usually been done by means of translation: the necessary precondition for the circulation of meaning on a global scale (Bielsa 2005: 139), especially in emergency situations requiring a rapid and smooth flow of information between people and institutions (Zhang and Wu 2020: 527). Overwhelmingly, the source language has been English, given its predominance in institutional and scientific settings such as the European Union (e.g. Biel et al. 2018: 251, Piller et al. 2020: 505, Seracini 2020: 1-2).

This study investigates how multi-word terms relating to the COVID-19 pandemic are rendered from English into Polish by employing a parallel corpus of EU press releases. To the best of the authors' knowledge, research into translation-mediated communication between EU institutions and the public during the pandemic has been limited, especially with respect to terminology. Furthermore, terminology in general is mostly studied in specialized language (Drouin et al. 2017), whereas little attention has been paid to terms when they "move out of their [specialized] sphere and participate in new forms of written and oral interactions" (Delavigne 2017: 32) such as expert-to-lay communication. The present study aims to fill these research gaps by employing quantitative approaches to the study of terminology which has also been recognized as overlooked in the literature (Biel and Koźbiał 2020). It will shed light on how multiword terms are handled in translation within the context of COVID-19 considering their potential neological instability, as well as the characteristics of EU expert-to-lay translation, and the constraints imposed by the pandemic.

Therefore, this study aims at answering the following questions: (1) How can COVID-19 terms employed in EU press releases be classified according to their thematic scope? (2) What is the degree of variation of equivalents in COVID-19 terms? (3) To what extent are their equivalents institutionalized in translations into Polish? (4) What is the degree of domesti- 
cation/foreignization of terminological equivalents of COVID-19 terms? (5) What is the degree of transfer of information from SL (Source Language) to TL (Target Language) equivalents?

\section{Context of the study}

\subsection{COVID-19 pandemic}

SARS-CoV-2 - the virus which causes the disease - was first identified in December 2019 in the Chinese municipality of Wuhan (Chaplin 2020). It soon crossed into neighboring countries and by the end of the following month had spread to other continents. The first case in Europe was reported in France on January 24, 2020; by March 2020, all EU member states were affected (Goniewicz et al. 2020: 3). On March 11, 2020, the World Health Organization (WHO) declared the COVID -19 outbreak to be a pandemic. As of October 26, 2020, more than 42 million infection cases had been diagnosed across the globe, with over a million deaths (WHO 2020).

Over eight million diagnoses had been confirmed in the European Union as of October 26 (European Centre for Disease Prevention and Control 2020). Propelled by the growing consequences of the pandemic, EU institutions have been forced to adopt relevant legislation and undertake measures so as to facilitate the flow of information, the assessment of needs, and the introduction of a consistent EU-wide response (Goniewicz et al. 2020). The latter has focused on a number of priorities, most importantly health (the adoption of preventive measures, the purchase of medical equipment and supplies) and research (developing a vaccine and treatment therapies), as well as various measures intended to curb the pandemic (e.g. travel restrictions) and to alleviate its effects on the economy (Goniewicz et al. 2020). Consequently, the role of translation (mostly from English to other languages, given the predominance of this language in institutional settings [Biel et al. 2018: 251, Piller et al. 2020: 505, Seracini 2020: 1-2]) as a key facilitator of com- 
munication between member states has grown exponentially. At the same time, the ongoing pandemic has exacerbated the need for accurate and fast translation-mediated communication between institutions and the general public in order to explain basic facts, risks, and ways to minimize them (Costa-Sánchez and López-García 2020).

\subsection{EU translation: press releases}

Out of the two types of communication observed in EU translation - expert-to-expert and expert-to-lay (Biel 2014: 56) - this study is concerned with the latter. Specifically, it examines a corpus of press releases. Press releases can be defined as:

relatively short texts resembling news stories and containing what is considered by the issuer to be newsworthy information; they are generally sent to the journalist community (but the intended primary readership has been recently shifting to the general public) with the purpose of having them picked up by the press and turned into actual news stories. (Catenaccio 2008: 13)

Thus, the primary function of a press release is to become transformed into news intended for the general public (Lassen 2006). As a result, this genre constitutes an important link in the (usually translator-mediated) transmission of knowledge from (EU) authorities and experts to the citizens.

The two categories of EU translation are governed by different rules and expectations with regard to quality. Whereas the highest quality is required from legal translation, where the notion of equivalence is foregrounded to ensure a uniform interpretation and application of EU law across member states (Biel 2017: 37 , texts directed at the general public are expected to be natural and readable above all else: "a key quality desideratum is to produce texts that read like originals in all languages" (Directorate-General for Translation 2015: 2,13). This is achieved by localizing translations to TL conventions, high idiomaticity, and 
avoiding EU jargon (Biel 2017: 38). In order to produce naturalsounding language, translators of texts addressed to laypeople also have more agency than those responsible for specialized communication (Biel 2017: 38).

\subsection{Terminology in translation}

The translation of texts containing terms, i.e. "lexical units with a precise meaning in a given special field" (Cabré 2010: 359), presents a challenge in translation, especially given their role of access points to knowledge structures (Faber 2009: 109). This process consists of bridging the gap between two - potentially incongruous - concepts (Biel 2009: 183, Hejwowski 2004: 15). The degree of their incongruity may vary - from (nearly) identical concepts (which are universal or, in institutional settings, supranational) to "conceptual voids" without any equivalent in the TL (Biel 2009: 183). The latter end of the spectrum is occupied in particular by system- and culture-specific items, for example legal terms (Sosoni and O'Shea 2020).

Similarly, neologisms might often pose challenges in translation. Their spread is associated with some of the more dynamically evolving fields like medicine (León-Araúz 2017: 215-216), as well as with political, economic, or social changes (Carter 1999, Seekowska 2002, Maybin and Swann 2007), such as the ongoing health crisis (Cierpich-Kozieł 2020, Lawson 2020). Closing the gap between these newly coined or modified terms might, therefore, require significant effort on the part of translators. Nevertheless, translators should ensure that terms in translation have precise and unambiguous meanings and that they are - ideally - always translated in the same manner to ensure terminological stability (Hejwowski 2004: 14). In the EU context, this consistency should be maintained both at the level of a given text, and with respect to other EU texts (Biel and Koźbiał 2020).

Despite the above-mentioned prescriptive view on terminology (Hejwowski 2004: 14), there is significant empirical evidence 
of terminological variation in specialized language. Term variation occurs "when different denominations are used to refer to the same concept" (León-Araúz 2017: 214). Even though some studies have demonstrated that institutional (EU included) translations of terms tend to be consistent (Fernández-Silva and Kerremans 2011, Kerremans 2017), the overwhelming majority argue that complete terminological standardization is very difficult to achieve as definitions and concept systems are never static, and synonymy and polysemy often occur in specialized language (Freixa 2002, Faber 2009, Freixa and Fernández-Silva 2017, León-Araúz 2017, Pimentel 2017), including EU translation (Biel et al. 2018, Mori 2018, Prieto Ramos and Morales 2019, Seracini 2020, Biel and Koźbiał 2020). Terminological variation can be attributed to numerous communicative and cognitive factors, such as the situational context of specialized communication, the translator's knowledge of the topic and its terminology, his or her expectations regarding the target text readers' knowledge, the availability of terminological resources, and translation policy (Faber 2009: 113, Fernández-Silva and Kerremans 2011: 332, Freixa and Fernández-Silva 2017: 176). In the EU context, terminological variation also results from interference, low termness of neologisms, the fragmentation of translation services, insufficient terminological resources, as well as translators' lack of systematicity in following guidelines, and inadequate knowledge of TL terminology (Prieto Ramos and Morales 2019: 107-108, Biel 2020: 13, Biel and Koźbiał 2020). In the Polish context, it may also be due to "the instability of the Polish Eurolect at its formative stage" (Biel and Koźbiał 2020). This may ultimately influence the representation and transfer of specialized knowledge (Faber 2009: 108, Cabré 2010: 358), and on a micro-level, the reception and adoption of specific terms into the TL (Prieto Ramos and Morales 2019). 


\subsection{Translating a pandemic}

Translation processes during the COVID-19 health crisis can be associated with significant challenges. The rapid spread of the virus across the globe has required an equally fast circulation of translation-mediated information (Costa-Sánchez and LópezGarcia 2020). As a result, translation processes have been governed by pace above all else (Zhang and Wu 2020: 527). Apart from time pressures, other constraints have been posed on translators during the COVID-19 crisis. The obligatory confinement which resulted in school closures has made it arguably more difficult to balance work and childcare. At the same time, some of the translators with young families may have been forced to work from home, often in confined spaces, which might have impacted on the outcome of translation processes (Strouther 2020). With respect to terminology, translators may have encountered difficulties due to the lack or inadequacy of reference sources such as dictionaries, glossaries, etc. Even though new multilingual entries are regularly being added the EU's database of terminology (Interactive Terminology for Europe 2020), registering new terms might sometimes be delayed in EU translation services as a result of the rapidly evolving epidemiological situation. All these factors may ultimately lead to increased challenges connected with the rendering of COVID-19-related terminology, as well as a higher terminological variation.

\section{Materials and research procedure}

The EU-COV is a parallel English-Polish corpus of press releases on the subject of the COVID-19 pandemic, extracted from the web archive of the European Commission (https://europa.eu/newsroom/press-releases/). It covers a period of five months - from the date on which the WHO declared the situation a global pandemic, March 11, 2020 to August 11, 2020. Texts were selected using the search term coronavirus and choosing specific dates on the website. Sixty-seven English- 
language texts and sixty-seven corresponding Polish-language texts were downloaded. The texts were aligned using LF Aligner, manually verified for noise, and uploaded into Sketch Engine (Kilgariff et al. 2014). The structure of the corpus is presented in Table 1 below.

\section{Table 1}

Structure of the EU-COV corpus

\begin{tabular}{|l|c|c|c|}
\cline { 2 - 4 } \multicolumn{1}{c|}{} & EN & PL & TOTAL \\
\hline Tokens & 62,006 & 60,301 & 122,307 \\
\hline Unique words & 5,218 & 8,838 & 14,056 \\
\hline Texts & 67 & 67 & 134 \\
\hline
\end{tabular}

Multi-word terms were identified by comparing EU-COV to enTenTen2015 using the keywords extraction function in Sketch Engine. The enTenTen2015 corpus was used because it is sufficiently large, thematically and linguistically varied, and comprises relatively recent data (Jakubíček et al. 2013). Only keywords related to COVID-19 having raw frequency scores higher than one were chosen for analysis. The parallel concordances of English and Polish texts were subsequently examined to retrieve the TL equivalents of the identified SL terms. Next, each concordance (extended to full sentences or paragraphs when necessary) was analyzed in order to examine the use of translation techniques at four different levels:

1. Terminological variation. Each SL term was paired with its equivalent or equivalents in translated texts in order to establish the degree of term variation.

2. The level of institutionalization. Each English term was verified in the EU's terminology database, the Interactive Terminology for Europe (IATE), to find out whether a Polish equivalent had been recommended. The recommended equivalent (in some cases there were several) was then compared with the term(s) employed by the translators of the analyzed press releases. The 
aim was to esta-blish to what extent EU guidelines are followed in the translation of press releases. An important note to bear in mind is that some press releases may have been published prior to the introduction of specific COVID-19 terms (especially neologisms) into the database. Entries in IATE are not dated, hence establishing a timeline was not possible. As a result, any conclusions regarding the translators' consistency in following terminological gui-delines of the EU can only be drawn tentatively and have to be treated with caution.

3. The level of foreignization/domestication. Four main translation techniques along the foreignization - domes-tication continuum were considered: borrowing ("reproducing the SL expression [in TL]" [Weston 1991: 26]), literal equivalent ("formal (lexical) equivalence at the level of either the word or higher units" [Weston 1991: 24]), descriptive equivalent (paraphrasing SL information in TL [Biel 2009: 185]), and functional equivalent ("using a TL expression which denotes the nearest equivalent concept" [Weston 1991: 21]).

4. The degree of transfer of information. Each concordance was analyzed in order to examine whether the information in the TL text was expanded (amplification), reduced (reduction), made more general (generalization) or more specific (particularization) with respect to SL; or whether the information in the two parallel concordances was conveyed to the same extent (correspondence). This part of the analysis was partly inspired by Molina and Hurtado Albir's (2002) classification of translation techniques. The analyzed levels are presented in Figure 1. 


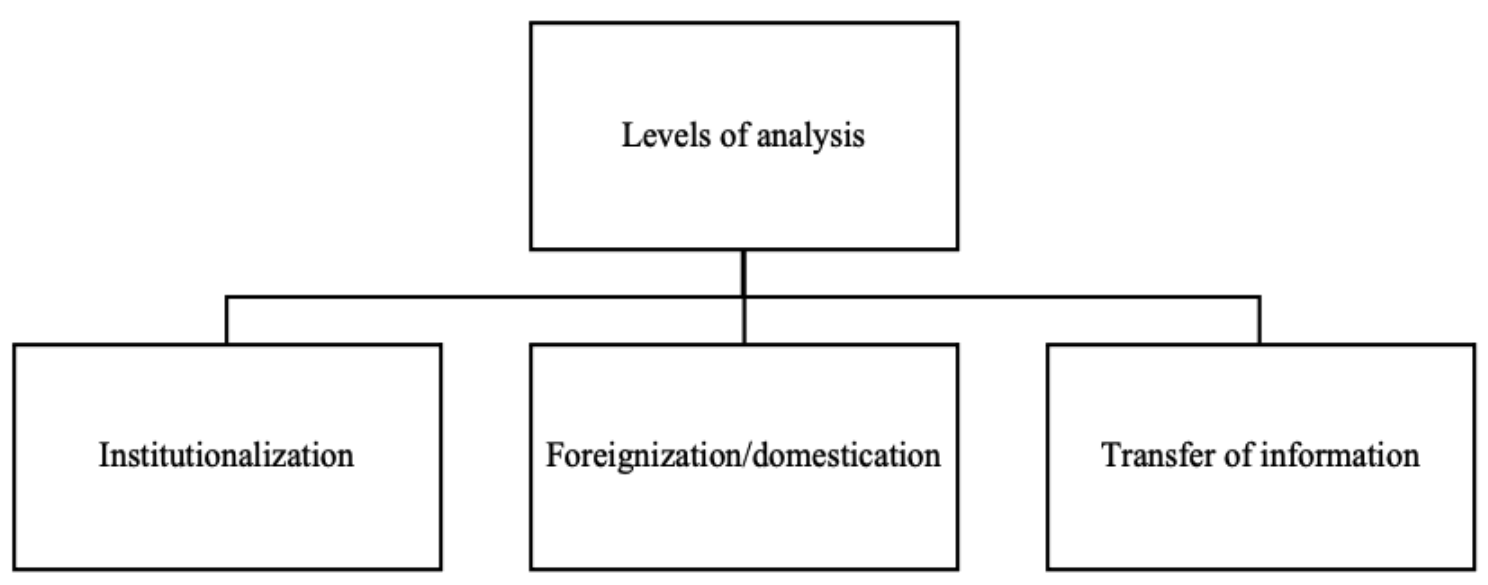

Figure 1

Levels of analysis of COVID-19 terms in EU-COV

\section{Results and discussion}

The identified terms are not thematically homogenous but can be grouped into three thematic categories: Health and research - 18 terms, Institutional response - 17 terms, and Protective measures -5 terms (40 terms in total):

(1) Health and research

coronavirus outbreak $(\mathrm{N}=87)$, coronavirus pandemic $(\mathrm{N}=56)$, medical equipment $(\mathrm{N}=23)$, cross-border healthcare $(\mathrm{N}=6)$, cross-border treatment $(\mathrm{N}=6)$, medical capacity $(\mathrm{N}=5)$, convalescent plasma $(\mathrm{N}=5)$, rapid point-of-care (diagnostic tests) $(\mathrm{N}=3)$, EU health preparedness $(\mathrm{N}=3)$, essential staff $(\mathrm{N}=3)$, large-scale testing $(\mathrm{N}=3)$, transmission chain $(\mathrm{N}=3)$, testing capacity $(\mathrm{N}=3)$, coronavirus emergency $(\mathrm{N}=2)$, expert capacity $(\mathrm{N}=2)$, respiratory equipment $(\mathrm{N}=2)$, mass testing $(\mathrm{N}=2)$, virus outbreak $(\mathrm{N}=2)$

(2) Institutional response

Temporary Framework $(\mathrm{N}=75)$, non-essential travel $(\mathrm{N}=33)$, travel restriction $(\mathrm{N}=40)$, supply chain $(\mathrm{N}=16)$, joint procurement $(\mathrm{N}=11)$, private storage aid $(\mathrm{N}=6)$, gradual lifting $(\mathrm{N}=6)$, general escape clause $(\mathrm{N}=5)$, industrial deployment $(\mathrm{N}=5)$, exit strategy $(\mathrm{N}=5)$, affected country $(\mathrm{N}=5)$, circular economy $(\mathrm{N}=2)$, screening framework $(\mathrm{N}=2)$, coordination hub 
$(\mathrm{N}=2)$, state aid register $(\mathrm{N}=2)$, interoperability solution $(\mathrm{N}=2)$, national stockpiling $(\mathrm{N}=2)$

(3) Protective measures

protective equipment $(\mathrm{N}=32)$, personal protective equipment $(\mathrm{N}=13)$, protective clothing $(\mathrm{N}=12)$, covid-19 confinement $(\mathrm{N}=10)$, social distancing $(\mathrm{N}=5)$

Parallel concordances were analyzed to pair SL terms with their TL equivalents. In sum, there are 89 pairs of SL-TL multi-word terms. Since the analysis reveals that the majority of the terms have more than one TL equivalent, terminological variation in the translations of the EU press releases is discussed briefly in the following section (4.1). It is followed by a discussion of the level of institutionalization of the employed TL terms (section 4.2), translation techniques on a scale from domestication to foreignization (section 4.3), and the degree of transfer of information (section 4.4). Selected SL terms for each thematic category, along with their equivalents and the results of the analyses, are presented in Tables 2, 3, and 4 below. Full data was published in an external database (Tomaszewska and Zawadzka-Paluektau 2020).

Table 2

Selected SL terms and their TL equivalents in COVID-19

EU press releases, category:

Health and research

\begin{tabular}{|c|c|c|c|c|c|}
\hline SL term & TL equivalent & $\mathrm{N}$ & $\begin{array}{l}\text { IATE } \\
\text { equiv- } \\
\text { alent }\end{array}$ & $\begin{array}{l}\text { Domesti- } \\
\text { cation / } \\
\text { foreigniza- } \\
\text { tion }\end{array}$ & $\begin{array}{l}\text { Transfer } \\
\text { of infor- } \\
\text { mation }\end{array}$ \\
\hline \multirow{2}{*}{$\begin{array}{l}\text { corona- } \\
\text { virus } \\
\text { outbreak } \\
(\mathrm{N}=87)\end{array}$} & koronawirus & 31 & -(new) & $\begin{array}{l}\text { functional } \\
\text { equivalent }\end{array}$ & reduction \\
\hline & $\begin{array}{l}\text { pandemia } \\
\text { [+ five other } \\
\text { equivalents] }\end{array}$ & 13 & -(new) & $\begin{array}{l}\text { functional } \\
\text { equivalent }\end{array}$ & $\begin{array}{l}\text { generaliza- } \\
\text { tion }\end{array}$ \\
\hline $\begin{array}{l}\text { corona- } \\
\text { virus }\end{array}$ & $\begin{array}{l}\text { pandemia ko- } \\
\text { rona-wirusa }\end{array}$ & 50 & $+($ new $)$ & $\begin{array}{l}\text { functional } \\
\text { equivalent }\end{array}$ & $\begin{array}{l}\text { corre- } \\
\text { spondence }\end{array}$ \\
\hline
\end{tabular}




\begin{tabular}{|c|c|c|c|c|c|}
\hline $\begin{array}{l}\text { pandemic } \\
(\mathrm{N}=56)\end{array}$ & $\begin{array}{l}\text { [+ three other } \\
\text { equivalents] }\end{array}$ & & & & \\
\hline \multirow{3}{*}{$\begin{array}{l}\text { medical } \\
\text { capacity } \\
(\mathrm{N}=5)\end{array}$} & $\begin{array}{l}\text { rezerwy } \\
\text { medyczne }\end{array}$ & 3 & \multirow{3}{*}{ (ø) } & $\begin{array}{l}\text { functional } \\
\text { equivalent }\end{array}$ & $\begin{array}{l}\text { corre- } \\
\text { spondence }\end{array}$ \\
\hline & $\begin{array}{l}\text { zdolność } \\
\text { medyczna }\end{array}$ & 1 & & $\begin{array}{l}\text { literal } \\
\text { equivalent }\end{array}$ & $\begin{array}{l}\text { corre- } \\
\text { spondence }\end{array}$ \\
\hline & rezerwy & 1 & & $\begin{array}{l}\text { functional } \\
\text { equivalent }\end{array}$ & $\begin{array}{l}\text { generaliza- } \\
\text { tion }\end{array}$ \\
\hline $\begin{array}{l}\text { convales- } \\
\text { cent } \\
\text { plasma } \\
(\mathrm{N}=5)\end{array}$ & $\begin{array}{l}\text { osocze } \\
\text { ozdrowieńców }\end{array}$ & 5 & $+($ new $)$ & $\begin{array}{l}\text { functional } \\
\text { equivalent }\end{array}$ & $\begin{array}{l}\text { corre- } \\
\text { spondence }\end{array}$ \\
\hline \multirow{2}{*}{$\begin{array}{l}\text { EU } \\
\text { health } \\
\text { prepar- } \\
\text { edness } \\
(\mathrm{N}=3)\end{array}$} & $\begin{array}{l}\text { gotowość UE } \\
\text { w dziedzinie } \\
\text { zdrowia }\end{array}$ & 2 & \multirow[t]{2}{*}{ (Ø) } & $\begin{array}{l}\text { descriptive } \\
\text { equivalent }\end{array}$ & $\begin{array}{l}\text { amplifica- } \\
\text { tion }\end{array}$ \\
\hline & gotowość UE & 1 & & $\begin{array}{l}\text { functional } \\
\text { equivalent }\end{array}$ & $\begin{array}{l}\text { generaliza- } \\
\text { tion }\end{array}$ \\
\hline \multirow{2}{*}{$\begin{array}{l}\text { essential } \\
\text { staff } \\
(\mathrm{N}=3)\end{array}$} & $\begin{array}{l}\text { pracownicy } \\
\text { kluczowi }\end{array}$ & 2 & $-($ new) & $\begin{array}{l}\text { literal } \\
\text { equivalent }\end{array}$ & $\begin{array}{l}\text { corre- } \\
\text { spondence }\end{array}$ \\
\hline & pracownicy & 1 & $-($ new) & $\begin{array}{l}\text { functional } \\
\text { equivalent }\end{array}$ & $\begin{array}{l}\text { generaliza- } \\
\text { tion }\end{array}$ \\
\hline $\begin{array}{l}\text { trans- } \\
\text { mission } \\
\text { chain } \\
(\mathrm{N}=3)\end{array}$ & $\begin{array}{l}\text { łańcuch } \\
\text { zakażeń }\end{array}$ & 3 & $+($ new $)$ & $\begin{array}{l}\text { functional } \\
\text { equivalent }\end{array}$ & $\begin{array}{l}\text { corre- } \\
\text { spondence }\end{array}$ \\
\hline $\begin{array}{l}\text { corona- } \\
\text { virus } \\
\text { emer- } \\
\text { gency } \\
(\mathrm{N}=2)\end{array}$ & $\begin{array}{l}\text { zagrożenie } \\
\text { koronawiru- } \\
\text { sem }\end{array}$ & 2 & $-($ new $)$ & $\begin{array}{l}\text { functional } \\
\text { equivalent }\end{array}$ & reduction \\
\hline \multirow[t]{2}{*}{$\begin{array}{l}\text { mass } \\
\text { testing } \\
(\mathrm{N}=2)\end{array}$} & $\begin{array}{l}\text { prowadzone } \\
\text { na masowa } \\
\text { skalę działa- } \\
\text { nia w zakresie } \\
\text { testowania }\end{array}$ & 1 & - & $\begin{array}{l}\text { descriptive } \\
\text { equivalent }\end{array}$ & $\begin{array}{l}\text { amplifica- } \\
\text { tion }\end{array}$ \\
\hline & $\begin{array}{l}\text { masowe } \\
\text { przeprowadza- } \\
\text { nie testów }\end{array}$ & 1 & - & $\begin{array}{l}\text { descriptive } \\
\text { equivalent }\end{array}$ & $\begin{array}{l}\text { amplifica- } \\
\text { tion }\end{array}$ \\
\hline
\end{tabular}


Explanation of symbols in the IATE equivalent column (applicable also to Tables 4 and 5): (ø) the SL term is not included in IATE as of October 6, 2020; + TL term is a recommended equivalent; - TL term is not a recommended equivalent; (new) the term was added during the COVID-19 pandemic (Interactive Terminology for Europe 2020). Other comments in brackets, e.g. (preferred), are recommendations of use provided by IATE.

Table 3

Selected SL terms and their TL equivalents in COVID-19 EU press releases, category: Institutional response

\begin{tabular}{|c|c|c|c|c|c|}
\hline SL term & TL equivalent & $\mathrm{N}$ & $\begin{array}{c}\text { IATE } \\
\text { equiva- } \\
\text { lent } \\
\end{array}$ & $\begin{array}{l}\text { Domestica- } \\
\text { tion/ for- } \\
\text { eignization }\end{array}$ & $\begin{array}{c}\text { Transfer } \\
\text { of infor- } \\
\text { mation } \\
\end{array}$ \\
\hline \multirow[b]{2}{*}{$\begin{array}{l}\text { Tempo- } \\
\text { rary } \\
\text { Frame- } \\
\text { work } \\
(\mathrm{N}=75)\end{array}$} & $\begin{array}{l}\text { tymczasowe } \\
\text { ramy }\end{array}$ & 44 & $+($ new $)$ & $\begin{array}{l}\text { functional } \\
\text { equivalent }\end{array}$ & $\begin{array}{l}\text { corre- } \\
\text { spondence }\end{array}$ \\
\hline & $\begin{array}{l}\text { tymczasowe } \\
\text { ramy pomocy } \\
\text { państwa } \\
\text { [+ three other } \\
\text { equivalents] }\end{array}$ & 19 & $+($ new $)$ & $\begin{array}{l}\text { functional } \\
\text { equivalent }\end{array}$ & $\begin{array}{l}\text { amplifica- } \\
\text { tion }\end{array}$ \\
\hline \multirow{2}{*}{$\begin{array}{l}\text { non-es- } \\
\text { sential } \\
\text { travel } \\
(\mathrm{N}=33)\end{array}$} & $\begin{array}{l}\text { inne niż } \\
\text { niezbędne } \\
\text { podróże }\end{array}$ & 32 & + & $\begin{array}{l}\text { descriptive } \\
\text { equivalent }\end{array}$ & $\begin{array}{l}\text { corre- } \\
\text { spondence }\end{array}$ \\
\hline & $\begin{array}{l}\text { podróże, które } \\
\text { są niezbędne }\end{array}$ & 1 & - & $\begin{array}{l}\text { descriptive } \\
\text { equivalent }\end{array}$ & $\begin{array}{l}\text { corre- } \\
\text { spondence }\end{array}$ \\
\hline $\begin{array}{l}\text { joint } \\
\text { pro- } \\
\text { cure- } \\
\text { ment } \\
(\mathrm{N}=11)\end{array}$ & $\begin{array}{l}\text { zamówienie } \\
\text { wspólne/ } \\
\text { wspólne } \\
\text { zamówienie }\end{array}$ & 6 & $+($ new $)$ & $\begin{array}{l}\text { functional } \\
\text { equivalent }\end{array}$ & $\begin{array}{l}\text { particu- } \\
\text { larization }\end{array}$ \\
\hline \multirow{2}{*}{$\begin{array}{l}\text { private } \\
\text { storage } \\
\text { aid } \\
(\mathrm{N}=6)\end{array}$} & $\begin{array}{l}\text { dopłaty do } \\
\text { prywatnego } \\
\text { przechowywa- } \\
\text { nia }\end{array}$ & 5 & + & $\begin{array}{l}\text { functional } \\
\text { equivalent }\end{array}$ & $\begin{array}{l}\text { corre- } \\
\text { spond- } \\
\text { ence }\end{array}$ \\
\hline & $\begin{array}{l}\text { srodki w for- } \\
\text { mie dopłat do }\end{array}$ & 1 & - & $\begin{array}{l}\text { descriptive } \\
\text { equivalent }\end{array}$ & $\begin{array}{l}\text { amplifica- } \\
\text { tion }\end{array}$ \\
\hline
\end{tabular}




\begin{tabular}{|c|c|c|c|c|c|}
\hline & $\begin{array}{l}\text { prywatnego } \\
\text { przechowywa- } \\
\text { nia }\end{array}$ & & & & \\
\hline $\begin{array}{l}\text { gradual } \\
\text { lifting } \\
(\mathrm{N}=6)\end{array}$ & $\begin{array}{l}\text { stopniowe } \\
\text { zniesienie/ } \\
\text { znoszenie }\end{array}$ & 6 & $+($ new $)$ & $\begin{array}{l}\text { functional } \\
\text { equivalent }\end{array}$ & $\begin{array}{l}\text { corre- } \\
\text { spond- } \\
\text { ence }\end{array}$ \\
\hline \multirow{2}{*}{$\begin{array}{l}\text { exit } \\
\text { strategy } \\
(\mathrm{N}=5)\end{array}$} & $\begin{array}{l}\text { strategia } \\
\text { wyjścia }\end{array}$ & 6 & $+($ new $)$ & $\begin{array}{l}\text { literal equiv- } \\
\text { alent }\end{array}$ & $\begin{array}{l}\text { corre- } \\
\text { spond- } \\
\text { ence }\end{array}$ \\
\hline & $\begin{array}{l}\text { strategia wyj- } \\
\text { ścia } z \text { kryzysu } \\
\text { [+ two other } \\
\text { equivalents] }\end{array}$ & 5 & $-($ new) & $\begin{array}{l}\text { descriptive } \\
\text { equivalent }\end{array}$ & $\begin{array}{l}\text { particu- } \\
\text { larization }\end{array}$ \\
\hline \multirow{3}{*}{$\begin{array}{l}\text { affected } \\
\text { country } \\
(\mathrm{N}=5)\end{array}$} & $\begin{array}{l}\text { państwo dot- } \\
\text { knięte klęską }\end{array}$ & 2 & \multirow{3}{*}{$(\varnothing)$} & $\begin{array}{l}\text { descriptive } \\
\text { equivalent }\end{array}$ & $\begin{array}{l}\text { amplifica- } \\
\text { tion }\end{array}$ \\
\hline & $\begin{array}{l}\text { kraj dotknięty } \\
\text { pandemia }\end{array}$ & 2 & & $\begin{array}{l}\text { descriptive } \\
\text { equivalent }\end{array}$ & $\begin{array}{l}\text { amplifica- } \\
\text { tion }\end{array}$ \\
\hline & kraj dotknięty & 1 & & $\begin{array}{l}\text { literal equiv- } \\
\text { alent }\end{array}$ & $\begin{array}{l}\text { corre- } \\
\text { spond- } \\
\text { ence }\end{array}$ \\
\hline \multirow{2}{*}{$\begin{array}{l}\text { screen- } \\
\text { ing } \\
\text { frame- } \\
\text { work } \\
(\mathrm{N}=2)\end{array}$} & wytyczne & 1 & \multirow[b]{2}{*}{$(\varnothing)$} & $\begin{array}{l}\text { functional } \\
\text { equivalent }\end{array}$ & $\begin{array}{l}\text { generali- } \\
\text { zation }\end{array}$ \\
\hline & $\begin{array}{l}\text { ramy monito- } \\
\text { rowania }\end{array}$ & 1 & & $\begin{array}{l}\text { literal equiv- } \\
\text { alent }\end{array}$ & $\begin{array}{l}\text { corre- } \\
\text { spond- } \\
\text { ence }\end{array}$ \\
\hline
\end{tabular}

Table 4

Selected SL terms and their TL equivalents in COVID-19 EU press releases, category: Protective measures

\begin{tabular}{|l|l|l|l|l|l|}
\hline SL term & $\begin{array}{l}\text { TL equiva- } \\
\text { lent }\end{array}$ & $\mathrm{N}$ & $\begin{array}{l}\text { IATE } \\
\text { equiva- } \\
\text { lent }\end{array}$ & $\begin{array}{l}\text { Domesti- } \\
\text { cation/ } \\
\text { foreigniza- } \\
\text { tion }\end{array}$ & $\begin{array}{l}\text { Transfer } \\
\text { of infor- } \\
\text { mation }\end{array}$ \\
\hline $\begin{array}{l}\text { protective } \\
\text { equip- } \\
\text { ment } \\
(\mathrm{N}=32)\end{array}$ & $\begin{array}{l}\text { środki } \\
\text { ochrony } \\
\text { [+ three } \\
\text { other }\end{array}$ & 23 & -(new) & $\begin{array}{l}\text { functional } \\
\text { equivalent }\end{array}$ & $\begin{array}{l}\text { corre- } \\
\text { spondence }\end{array}$ \\
\hline
\end{tabular}




\begin{tabular}{|c|c|c|c|c|c|}
\hline & equivalents] & & & & \\
\hline $\begin{array}{l}\text { protective } \\
\text { clothing } \\
(\mathrm{N}=12)\end{array}$ & $\begin{array}{l}\text { odzież } \\
\text { ochronna }\end{array}$ & 12 & + & $\begin{array}{l}\text { functional } \\
\text { equivalent }\end{array}$ & $\begin{array}{l}\text { corre- } \\
\text { spondence }\end{array}$ \\
\hline \multirow{2}{*}{$\begin{array}{l}\text { social } \\
\text { distanc- } \\
\text { ing }(\mathrm{N}=5)\end{array}$} & $\begin{array}{l}\text { ograniczenie } \\
\text { kontaktów } \\
\text { osobistych }\end{array}$ & 2 & $+($ new $)$ & $\begin{array}{l}\text { descriptive } \\
\text { equivalent }\end{array}$ & $\begin{array}{l}\text { corre- } \\
\text { spondence }\end{array}$ \\
\hline & $\begin{array}{l}\text { ograniczenie } \\
\text { kontaktów } \\
\text { personal- } \\
\text { nych }\end{array}$ & 3 & $+($ new $)$ & $\begin{array}{l}\text { descriptive } \\
\text { equivalent }\end{array}$ & $\begin{array}{l}\text { corre- } \\
\text { spondence }\end{array}$ \\
\hline
\end{tabular}

\subsection{Terminological variation}

Variation in terminology can be defined as "diverging use of terms within one language" (Humbley and Picton 2017: 6). Sixty-five percent of COVID-19 terms extracted from the EUCOV corpus display interlinguistic variation, i.e. variation resulting from contact between languages (León-Araúz 2017: $215)$. This finding is in line with previous contributions to the study of interlinguistic variation (Pimentel 2017, Rossi 2017), including in the EU context (Biel et al. 2018, Mori 2018, Prieto Ramos and Morales 2019, Seracini 2020, Biel and Koźbiał 2020). As shown in Table 5, the category of Health and research displays the highest variation among the three categories: $72 \%$ of all words in this category have more than one lexicalization in the translated text. The categories of Institutional response and Protective measures, on the other hand, exhibit similar terminological variation levels: $58 \%$ and $60 \%$, respectively. 
Table 5

Terminological variation in COVID-19 press releases

\begin{tabular}{|l|c|c|c|}
\hline \multicolumn{1}{|c|}{ Category } & $\begin{array}{c}\text { Terms with one } \\
\text { TL equivalent }\end{array}$ & $\begin{array}{c}\text { Terms with more than } \\
\text { one TL equivalent }\end{array}$ & Total \\
\hline $\begin{array}{l}\text { Health and re- } \\
\text { search }\end{array}$ & $5(28 \%)$ & $13(72 \%)$ & 18 \\
\hline $\begin{array}{l}\text { Institutional } \\
\text { response }\end{array}$ & $7(41 \%)$ & $10(59 \%)$ & 17 \\
\hline $\begin{array}{l}\text { Protective } \\
\text { measures }\end{array}$ & $2(40 \%)$ & $3(60 \%)$ & 5 \\
\hline
\end{tabular}

All three categories follow the same pattern evident in the most frequent, and, therefore, the most salient SL terms whilst at the same time displaying the highest level of variation. These two dimensions - salience and variation - coincide in the two most frequently used terms in the category of Health and research: coronavirus outbreak and coronavirus pandemic (seven and four variants, respectively), as well as in the most frequently used terms in the other two categories: Temporary Framework (five variants) and protective equipment (four variants).

The significant level of interlinguistic variation of terms relating to COVID-19 in EU press releases may be attributed to a variety of communicative and cognitive factors. First, translation processes during the pandemic have been constrained by a variety of factors, such as time pressure, working conditions during lockdown, as well as insufficient guidelines and terminological resources, as discussed in section 2.4.

Secondly, terminological variation might be deliberately used as a strategy to convey information or help advance an argument by bringing out specific aspects of a given concept (Freixa and Fernández-Silva 2017: 176, Humbley and Picton 2017: 6). For example, the three variants of the term testing capacity focus either on the EU capacity to conduct tests ['zdolność przeprowadzania testów'], the availability of tests ['szeroka dostępność testów'], or the number of performed tests ['liczba wykonywanych testów'], thus foregrounding different aspects of 
the same concept. This may sometimes be connected to translators' expectations of the audiences' knowledge (Freixa and Fernández-Silva 2017: 176) and is particularly common with respect to new terms (Humbley and Picton 2017: 6).

In fact, terminological variation was observed in neologisms in particular. As expected (León-Araúz 2017: 215-216), medical terms were revealed to be particularly prone to terminological instability due to the dynamic developments within the field triggered by the health crisis (most notably in the two most frequent terms: coronavirus outbreak and coronavirus pandemic). This instability, however, might, in this context, be seen as a phase rather than a permanent feature of COVID-19 terminology.

Thirdly, terminological variation in EU press releases may result from the properties of the genre under study. As discussed above, EU translators of texts addressed to the general public are granted more agency than those responsible for specialized communication (Biel 2017: 38). Therefore, terminological consistency is not required in press releases to the same extent as in legal genres. Furthermore, in translations into Polish, repetition might be avoided due to its association with a poor style.

\subsection{The level of institutionalization}

The second step of the analysis was concerned with the level of institutionalization of the analyzed terms in translation into Polish. It aimed at establishing whether the TL terms are equivalents recommended by EU institutions. However, it is important to note that one-third of the SL COVID-19 terms extracted from the press releases analyzed ( $33 \%$, i.e. 13 terms) do not have their TL equivalents in IATE (as of October 6, 2020). This might be explained, above all, by their low termness (Shelov 2018: 48), as in the following examples: affected country, medical capacity, virus outbreak, and EU health preparedness. Furthermore, some of these terms - EU health preparedness, for instance - are likely to have gained significant salience only recently, during the pandemic, which might also explain their 
absence from the database. Other terms, however, are not included in IATE despite their high termness (Shelov 2018: 48), for example, screening framework and cross-border treatment. This indicates the incompleteness of the EU's terminological resource and the resulting lack of guidelines with respect to some terms.

The remainder of the SL terms identified in this study (27 terms, $68 \%$ ) are included in IATE. In the analyzed press releases, they have, in total, 61 TL equivalents. Out of these 61 terms, however, only 28 (46\%) are recommended equivalents. These include, first, terms included in the database before the pandemic, such as the TL equivalents of protective clothing; and second, terms which have become functional or established equivalents during the coronavirus crisis as a result of their significant salience in discourses surrounding the COVID-19 pandemic, e.g. coronavirus outbreak, non-essential travel, and gradual lifting. In fact, the vast majority of the analyzed recommended TL equivalents were also recognized as functional equivalents (22 terms, $79 \%)$. Third, the database includes terms with a significant degree of termness (Shelov 2018: 48), e.g. Temporary Framework and private storage aid.

Thirty-three TL terms are not among the established equivalents recommended by IATE. However, it is important to bear in mind that some of the press releases analyzed might have been published before specific terms were added to the database. This may result from the fact that the body of knowledge on COVID-19 has been growing systematically and rapidly, and the various measures to curb the pandemic or to alleviate its effects have been adopted on a short-term basis. New terms, associated with specific aspects of the crisis, were coined and entered into institutional or wide usage, and old terms became more salient within very short periods of time. In fact, 458 new multilingual entries related to the SARS-CoV-2 virus and the COVID-19 pandemic had been added to the database by July 28, including 15 terms analyzed in this study (marked as "new" in Tables 3-5), 
e.g. convalescent plasma, essential staff, and social distancing (Interactive Terminology for Europe 2020).

However, as entries in IATE are not dated, it is impossible to ascertain whether the terms which are not recommended equivalents were not in the database at the time of writing of a given press release, or whether the translator for some reason did not follow the existing guidelines. First, translators might have failed to look up terms displaying a low level of termness (Shelov 2018: 48). For example, the term testing capacity had three different variants in translation, none of which followed the IATE recommendation: zdolność testowania ['testing capacity']. In a similar way, essential staff, for which IATE suggests several synonymous equivalents (e.g. pracownicy o krytycznym znaczeniu ['staff of critical importance'] and niezbędni pracownicy ['indispensable staff']), was translated either as pracownicy kluczowi ['key staff'] or using the general word pracownicy ['staff']. Consequently, both TL equivalents provide much fewer specific renderings of the source term.

The second reason for not following IATE's recommendations might be genre-specific. In EU translation of texts addressed to the general public, there is significantly less emphasis on the equivalence of terms than in specialized communication. Instead, texts directed at the general public are expected to be natural and readable above all else. As a consequence, much more agency is granted to translators of expert-to-lay texts who might therefore opt for translation techniques that increase readability instead of resorting to established equivalents. For example, IATE's recommended Polish equivalent for coronavirus emergency is sytuacja nadzwyczajna zwiazana $z$ koronawirusem ['exceptional situation related to the coronavirus']. Instead of using the long and clumsy recommended equivalent, the translator(s) opted for a much shorter and natural-sounding zagrożenie koronawirusem ['coronavirus threat'] (in spite of the slight reduction in meaning).

For the same reason, translators also tended to decrease the level of termness of particular TL equivalents, therefore reducing 
the cognitive effort required from the readers. For example, in lieu of the recommended badanie przesiewowe populacji ['screenings test of the population'] as an equivalent for mass testing, translators avoided the highly specialized word przesiewowe ['screening'] and instead adopted more descriptive and explanatory translation solutions: prowadzone na masowa skale działania $w$ zakresie testowania ['activities with respect to testing conducted on a mass scale'] and masowe przeprowadzanie testów ['mass conducting of tests'].

Thirdly, non-adherence to terminological guidelines may be related to avoiding repetition in Polish, as it is regarded as poor style. For this reason, translators might sometimes resort to synonyms of recommended equivalents instead of invariably following the guidelines. Time pressures and other constraints which have affected the work of translators during the pandemic might also have played a role in limiting their reliance on terminological databases.

\subsection{Domestication - foreignization}

The third step in this analysis of translation techniques aimed at establishing the level of their foreignization/domestication. In this part of the study, such techniques as borrowings, literal equivalents, descriptive equivalents, as well as functional equivalents were identified and analyzed in SL-TL equivalents and concordances. Three out of the four translation techniques along the foreignization-domestication continuum were identified in the EU-COV corpus. The findings for each category and for the entire corpus are presented in Table 6. 
Table 6

Domestication/foreignization techniques per thematic category

\begin{tabular}{|l|c|c|c|c|}
\hline $\begin{array}{c}\text { Translation } \\
\text { technique }\end{array}$ & $\begin{array}{c}\text { Health and } \\
\text { research }\end{array}$ & $\begin{array}{c}\text { Institutional } \\
\text { response }\end{array}$ & $\begin{array}{c}\text { Protective } \\
\text { measures }\end{array}$ & Total \\
\hline $\begin{array}{l}\text { functional } \\
\text { equivalent }\end{array}$ & $29(73 \%)$ & $18(44 \%)$ & $7(70 \%)$ & $54(61 \%)$ \\
\hline $\begin{array}{l}\text { descriptive } \\
\text { equivalent }\end{array}$ & $8(20 \%)$ & $12(31 \%)$ & $3(30 \%)$ & $23(26 \%)$ \\
\hline $\begin{array}{l}\text { literal equiv- } \\
\text { alent }\end{array}$ & $3(8 \%)$ & $8(21 \%)$ & $0(0 \%)$ & $11(12 \%)$ \\
\hline $\begin{array}{l}\text { not recognized } \\
\text { (typograph- } \\
\text { ical error) }\end{array}$ & $0(0 \%)$ & $1(3 \%)$ & $0(0 \%)$ & $1(1 \%)$ \\
\hline
\end{tabular}

Domestication techniques are prevalent in COVID-19 press releases, with $61 \%$ functional and $26 \%$ descriptive equivalents in the material analyzed. With respect to the dominant technique of functional equivalent, its frequent use may attest to the presence of equivalents close to SL concepts which can be used in TL instead of coining new words or influencing SL meanings (in other words, introducing calques, borrowings, semantic extensions). This technique was most often applied in the Health and research category, where it appeared in $73 \%$ of the identified cases, and in Protective measures (70\%), whereas in the Institutional response category it was observed in only $44 \%$ of TL equivalents.

On the one hand, the identified functional equivalents include terms which were coined before the pandemic, e.g. łańcuch zakażeń ['transmission chain'] or osocze ozdrowieńców ['convalescent plasma']. On the other hand, the study reveals that equivalents of some of the terms coined during the pandemic have already become functional. Examples include new concepts related to alleviating the effects of the pandemic: Temporary Framework ['tymczasowe ramy'] and gradual lifting ['stopniowe zniesienie' or 'stopniowe znoszenie']. 
The second most commonly used technique on the domestication/foreignization scale is descriptive equivalent, which was observed in over a quarter (26\%) of all SL-TL equivalents: about $30 \%$ in both Institutional response and Protective measures, and $20 \%$ in Health and research (as the latter category is dominated to a greater extent by functional equivalents). It can be speculated that translators use this technique to avoid potential interpretation difficulties as it makes the TL equivalent more explicit by resolving text-inherent ambiguity (Biel 2009: 185). Moreover, it can provide "more (but not complete) information than the literal equivalent" (2009: 185). An example of a descriptive equivalent in EU-COV is the term non-essential travel rendered in TL as inne niż niezbędne podróże ['journeys other than necessary'] and podróże, które nie sa niezbędne ['journeys that are not necessary'].

In the material under review, foreignizing techniques were rarely diagnosed - the study revealed, on one hand, zero techniques resulting in non-integrated borrowings in TL, while on the other, relatively few literal equivalents (12\% among all SLTL equivalents). This may be due to the fact that EU press releases are part of non-expert communication, which is why translators may have chosen to bring the concepts closer to the non-specialist reader through domestication.

With respect to literal equivalents, these were observed in $8 \%$ of SL-TL equivalents in the Health and research category and in $21 \%$ in the Institutional response. Therefore, the present analysis indicates that this is not the preferred technique in EU translation of press releases, which might testify to the linguistic accuracy and expertise of translators of EU COVID-19 press releases. It might also be due to the nature of press releases as texts directed at wider audiences, which - contrary to expert-toexpert EU communication (legal texts in particular), where translation is expected to be as close to the original as possible - should rather aim at bringing the text closer to the reader, according to EU translation guidelines (Directorate-General for Translation 2015: 2, 13). What is more, in the few instances 
where literal equivalents were employed, the overwhelming result (10 out of 11 TL equivalents) was the creation of unnaturalsounding or even ungrammatical constructions in Polish (with the obvious exception of literal equivalents which have become functional). Examples include terms such as zdolność medyczna ['medical capacity'], strategia wyjścia ['exit strategy'], and kraj dotknięty ['affected country'].

\subsection{The degree of transfer of information}

The aim of the last part of the study was to examine whether and to what extent, when compared to the SL, the information in the TL was expanded (amplification), reduced (reduction), made more general (generalization), more specific (particularization) or conveyed to the same extent (correspondence). The findings are presented in Table 7.

Table 7

Transfer of information techniques per thematic category

\begin{tabular}{|l|c|c|c|c|}
\hline $\begin{array}{c}\text { Transfer of infor- } \\
\text { mation technique }\end{array}$ & $\begin{array}{c}\text { Health } \\
\text { and } \\
\text { research }\end{array}$ & $\begin{array}{c}\text { Institu- } \\
\text { tional } \\
\text { response }\end{array}$ & $\begin{array}{c}\text { Protective } \\
\text { measures }\end{array}$ & Total \\
\hline correspondence & $14(35 \%)$ & $17(44 \%)$ & $9(90 \%)$ & $40(45 \%)$ \\
\hline amplification & $8(20 \%)$ & $12(31 \%)$ & $1(10 \%)$ & $21(24 \%)$ \\
\hline generalization & $12(30 \%)$ & $2(5 \%)$ & $0(0 \%)$ & $14(16 \%)$ \\
\hline particularization & $3(7,5 \%)$ & $5(13 \%)$ & $0(0 \%)$ & $8(9 \%)$ \\
\hline reduction & $3(7,5 \%)$ & $2(5 \%)$ & $0(0 \%)$ & $5(6 \%)$ \\
\hline $\begin{array}{l}\text { not recognized } \\
\text { (typographical } \\
\text { error })\end{array}$ & $0(0 \%)$ & $1(3 \%)$ & $0(0 \%)$ & $1(1 \%)$ \\
\hline
\end{tabular}


SL information is most often transferred in EU-COV by means of correspondence ( $45 \%$ of cases), i.e. information from SL is transferred to TL to the same degree in less than half of the identified SL-TL equivalents. However, although it is the most commonly used technique in our material, the other techniques - which alter the degree of transfer of information - are predominant in the corpus. In other words, in most cases, the translated terms do not fully correspond to the source terms.

The highest level of correspondence was observed in the thematic category of Protective measures: $90 \%$ of the SL-TL equivalents. The high percentage of the use of the correspondence technique in this category is probably due to the prevalence of, on the one hand, technical and highly terminological items (which are, therefore, easily identified as terms), and, on the other, terminology already in use before the pandemic (which constitutes unambiguous references to the given concepts), for example protective clothing translated as odzież ochronna. In the remaining categories, the percentage is significantly lower $35 \%$ in Health and research and $44 \%$ in Institutional response, meaning that with respect to the majority of terms in both categories the translator chose techniques that somehow modify the degree of transfer of information. Correspondence is very often found in literal equivalents $(73 \%)$ but to a much lesser extent in functional equivalents (48\%). Correspondence was rarely achieved when descriptive equivalents were employed (22\%).

One of the techniques leading to interference in the transmission of information in TL is amplification. Translators may resort to this technique in order to manage possible reading and interpretation difficulties as the use of this technique makes the TL equivalent more explicit by resolving text-inherent ambiguity. Amplification was employed in $24 \%$ of the SL-TL equivalents in the whole research material - in $20 \%$ of the analyzed items in the category Health and research, in $31 \%$ in Institutional response, and in $10 \%$ in Protective measures. The terms in the latter thematic category do not require clarification (as 
mentioned above, their equivalents were used prior to the pandemic). In the other thematic categories, however, a number of new terms were used, prompting the use of amplification in order to clarify their meanings. For instance, Temporary Framework has five TL equivalents in the analyzed material, four of which are amplified. Amplification was most frequently used with descriptive equivalents $(71 \%)$, which indicates that whenever the translator opted for description as a translation technique, it often required the amplification of meaning of TL terms with respect to SL terms.

Another technique used was generalization, which results in the omission of some aspects of a concept due to the use of a more general or neutral TL term (Molina and Hurtado Albir 2002: 510). Generalization occurred in $16 \%$ of the entire research material, $30 \%$ in Health and research and $5 \%$ in Institutional response. It was not observed in the category Protective measures. For example, generalization was identified in the corpus in as many as five equivalents of the term coronavirus outbreak. In one of them, the words coronavirus and outbreak were omitted, and the term was translated as: pandemia ['pandemic']. Another example is EU health preparedness rendered as gotowość UE ['EU preparedness'] - here, the translator omitted the word health. The use of generalization as a translation technique results in a partial loss or blurring of the SL meaning.

The fourth most frequent transfer of information technique in the EU-COV corpus is particularization (9\%), which consists in making the information in TL more specific with respect to SL (Molina and Hurtado Albir 2002: 510). The use of this technique is rare in all thematic categories - it was used in $13 \%$ of cases in Institutional response, $9 \%$ in Protective measures, and $8 \%$ in Health and research. Particularization is usually observed in functional equivalents (63\% of all examples of particularization are functional equivalents), which may indicate that their contexts in Polish required narrower meanings. For instance, joint procurement has no exactly corresponding rendering available in TL as Polish equivalents refer to more specific contexts. 
Particularization, which was employed in four out of six TL equivalents of joint procurement, thus makes the TL texts less ambiguous by referring to one of several meanings or aspects of meanings of the SL term.

The transfer of information technique that is used least frequently in EU-COV (only $6 \%$ of the analyzed SL-TL equivalents) is reduction. Reduction consists in narrowing information in TL with respect to SL (Molina and Hurtado Albir 2002: 10). In other words, one or several aspects of an SL term are omitted in translation, which does not, however, lead to using a more general term, as in the case of generalization (which also results in a partial loss of meaning). In the focus corpus, reduction was used, for example, in the translation of the term exit strategy as wyjscie, where the word strategy was omitted in the TL text, and in coronavirus outbreak translated as koronawirus, where the TL element outbreak was omitted.

\section{Conclusions}

This study set out to investigate how multi-word terms relating to the COVID-19 pandemic are rendered in the translation of expert-to-lay communication from English into Polish. Therefore, it contributes to the study of terminology in non-specialized settings as well as to the advancement of the nascent branch of knowledge regarding the pandemic from a linguistic perspective.

The analysis shows that the COVID-19 multi-word terms can be divided into three main thematic categories. This indicates that EU communication directed at the general public focused on three main aspects of the COVID-19 crisis: the question of health and medical research, the institutional measures to curb the spread of the pandemic and alleviate its effects, as well as personal protective measures. A significant degree of overlap between national and supranational terminology has been observed in the corpus. 
Several findings suggest that terminology regarding the disease has not (yet) become fully stabilized. First, there is a significant degree of terminological variation. Sixty-five percent of the identified SL terms have more than one variant in TL. Secondly, a third of the extracted SL terms are not included in the EU's terminological database, and the existing guidelines are followed in less than half of the identified TL equivalents. Thirdly, with respect to the transfer of information, in the majority of terms, the techniques which alter the degree of transfer of information are employed instead of correspondence. Terminological instability was, above all, observed in neologisms. It might also be associated with the constraints that have been imposed on translators during the pandemic, and with the reduced levels of termness of the identified multi-words.

The characteristics and requirements of the genre analyzed, too, are likely to have affected the choice of translation strategies. First, terminological variation may result from stylistic concerns or different assumptions that translators have of their lay audiences' knowledge. This is also associated with the fact that translators of non-specialized texts are granted more agency than translators of legal documents, and thus are not invariably obliged to use recommended equivalents. Secondly, with respect to the level of institutionalization of the analyzed terms, translations directed at the general public are expected to be natural and readable above all else, which might lead translators to rely less on terminological databases than in the case of legal documents, especially in cases where low or high levels of termness can be observed. Thirdly, the use of techniques which alter the degree of transfer of information as well as the use of domesticating techniques might also be due to the translators' efforts to make TL texts more readable and easier to understand for target lay audiences. The limited use of literal equivalents and avoidance of borrowings, on the other hand, might attest to linguistic accuracy or apparent expertise of translators of EU COVID-19 press releases. 
The findings of this study, however, have to be seen in light of potential limitations. On the one hand, the focus corpus comprises press releases published in the first five months of the pandemic, whereas terminology has, naturally, continued evolving. At the time of writing this paper, the world is in the ninth month of the pandemic. New terminology is continually introduced to account for the advancements in medical research regarding the new disease and the measures adopted at national and supranational levels. With respect to the EU, its translation bodies are bound to continue working on bringing the terminological databases up to date, especially with respect to COVID19 terminology. Future research should thus account for these developments.

On the other hand, more conclusive results with regard to how terminology is handled in expert-to-lay translation could be drawn if the focus corpus was compared to a corpus of specialized texts, for instance, EU legal documents. Such a comparative study would allow for attributing specific translation choices to factors related to genre more accurately. Future contributions might aim to fill this gap.

\section{References}

Biel, Łucja (2009). "Organization of background knowledge structures in legal language and related translation problems". Comparative Legilinguistics: International Journal for Legal Communication 1: 176-189.

Biel, Eucja (2014). Lost in the Eurofog: The Textual Fit of Translated Law. Frankfurt am Main: Peter Lang.

Biel, Eucja (2017). "Quality in institutional EU translation: Parameters, policies and practices". In: Tomáš Svoboda, Łucja Biel, Krzysztof Łoboda (eds.). Quality Aspects in Institutional Translation. Berlin: Language Science Press, 31-57.

Biel, Lucja (2020). "Eurolects and EU legal translation". In: Meng Ji, Sara Laviosa (eds.). The Oxford Handbook of Translation and Social Practices. Oxford: Oxford University Press, 1-25. 
Biel, Łucja, Agnieszka Biernacka, Anna Jopek-Bosiacka (2018). "Collocations of terms in EU competition law: A corpus analysis of EU English collocations". In: Silvia Marino, Łucja Biel, Martina Bajčić, Vilelmini Sosoni (eds.). Language and Law: The Role of Language and Translation in EU Competition Law. Springer, 249-274.

Biel, Łucja, Dariusz Koźbiał (2020). "How do translators handle (near-) synonymous legal terms? A mixed-genre parallel corpus study into the variation of EU English-Polish competition law terminology". Estudios de Traducción 10: 69-90.

Bielsa, Esperança (2005). "Globalisation and translation: A theoretical approach". Language and Intercultural Communication 5/2: 131144.

Cabré, M. Teresa (2010). "Terminology and translation". In: Yves Gambier, Luc van Doorslaer (eds.). Handbook of Translation Studies. Amsterdam - Philadelphia: John Benjamins, 356-365.

Carter, Ronald (1999). "Common language: Corpus, creativity and cognition". Language and Literature 8/3: 195-216.

Catenaccio, Paola (2008). "Press releases as a hybrid genre: Addressing the informative/promotional conundrum". Pragmatics 18/1: 9-31.

Chaplin, Steve (2020). "COVID-19: a brief history and treatments in development”. Prescriber 31/5: 23-28.

Cierpich-Kozieł, Agnieszka (2020). "Koronarzeczywistość - o nowych złożeniach $\mathrm{z}$ członem korona- $\mathrm{w}$ dobie pandemii”. Język Polski 4. Available at https://jezyk-polski.pl/wp-content/uploads/2020/09 / Artyku\%C5\%82-Cierpich-Koziel.pdf. Accessed 26.10.2020.

Costa-Sánchez, Carmen, Xosé López-García (2020). "Comunicación y crisis del coronavirus en España. Primeras lecciones”. El Profesional de la Información 29/3: e290304.

Delavigne, Valérie (2017). "Term usage and socioterminological variation: The impact of social and local issues on the movement of terms". In: Patrick Drouin, Aline Francœur, John Humbley, Aurélie Picton (eds.). Multiple Perspectives on Terminological Variation. Amsterdam, Philadelphia: John Benjamins, 31-56.

Directorate-General for Translation, European Commission (2015). DGT Translation Quality Guidelines. Available at https://ec.europa. $\mathrm{eu} /$ translation/maltese/guidelines/documents/dgt_translation_q uality_guidelines_en.pdf. Accessed 08.09.2020. 
Drouin, Patrick, Aline Francœur, John Humbley, Aurélie Picton (2017). Multiple Perspectives on Terminological Variation. Amsterdam - Philadelphia: John Benjamins.

European Centre for Disease Prevention and Control (2020). COVID19 situation update worldwide, as of 26 October 2020. Available at https://www.ecdc.europa.eu/en/geographical-distribution-2019ncov-cases. Accessed 26.10.2020.

European Commission. The Press Corner. Available at https://ec.europa.eu/commission/presscorner/home/en. Accessed 07.09.2020.

Faber, Pamela (2009). "The cognitive shift in terminology and specialized translation". MonTi: Monografias de Traducción e Interpretación 1: 107-134.

Fernández-Silva, Sabela, Koen Kerremans (2011). "Terminological variation in source texts and translations: A pilot study". Meta 56/2: 318-335.

Freixa, Judit (2002). La Variació terminològica: Anàlisi de la variació denomitiva en textos de different grau d'especialització de l'àrea de medi ambient. Barcelona: Institut Universitari de Linguística Aplicada/ Universitat Pompeu Fabra.

Freixa, Judit, Sabela Fernández-Silva (2017). "Terminological variation and the unsaturability of concepts". In: Patrick Drouin, Aline Francœur, John Humbley, Aurélie Picton (eds.). Multiple Perspectives on Terminological Variation. Amsterdam - Philadelphia: John Benjamins, 155-180.

Goniewicz, Krzysztof, Amir Khorram-Manesh, Attila J. Hertelendy, Mariusz Goniewicz, Katarzyna Naylor, Frederick M. Burkle Jr. (2020). "Current response and management decisions of the European Union to the COVID-19 outbreak: A review". Sustainability 12/9, 3838. Available at https://www.mdpi.com/2071-1050/12/ 9/3838/htm. Accessed 09.09.2020.

Hejwowski, Krzysztof (2004). Kognitywno-komunikacyjna teoria przekładu. Warszawa: Wydawnictwo Naukowe PWN.

Humbley, John, Aurélie Picton (2017). "Multiple perspectives on terminological variation". In: Patrick Drouin, Aline Francœur, John Humbley, Aurélie Picton (eds.). Multiple Perspectives on Terminological Variation. Amsterdam, Philadelphia: John Benjamins, 1-10.

Interactive Terminology for Europe (2020). COVID-19 Terminology Available in IATE (Data as of 28/07/2020). Available at https://data.europa.eu/euodp/en/data/dataset/covid-19-multili 
ngual-terminology-on-iate/resource/44522e37-6b1e-40be-aae2-9

4d5ca2b8e13. Accessed 12.10.2020.

Jakubíček, Miloš, Adam Kilgariff, Vojtěch Kovář, Pavel Rychlý, Vit Suchomel (2013). "The TenTen corpus family". 7th International Corpus Linguistics Conference CL, 125-127.

Kerremans, Koen (2017). "Towards a resource of semantically and contextually structured term variants and their translations". In: Patrick Drouin, Aline Francœur, John Humbley, Aurélie Picton (eds.). Multiple Perspectives on Terminological Variation. Amsterdam - Philadelphia: John Benjamins, 83-108.

Kilgariff, Adam, Vit Baisa, Jan Bušta, Miloš Jakubíček, Vojtěch Kovář, Jan Michelfeit, Pavel Rychlý, Vit Suchomel (2014). "The Sketch Engine: ten years on". Lexicography 1: 7-36.

Lassen, Inger (2006). "Is the press release a genre? A study of form and content". Discourse Studies 8/4: 503-530.

León-Araúz, Pilar (2017). "Term and concept variation in specialized knowledge dynamics". In: Patrick Drouin, Aline Francœur, John Humbley, Aurélie Picton (eds.). Multiple Perspectives on Terminological Variation. Amsterdam - Philadelphia: John Benjamins, 213258.

Lawson, Robert (2020). "Coronavirus has led to an explosion of new words and phrases - and that helps us cope". The Conversation. Available at https://theconversation.com/coronavirus-has-led-toan-explosion-of-new-words-and-phrases-and-that-helps-us-cope136909. Accessed 09.09.2020.

Maybin, Janet, Joan Swann (2007). "Everyday creativity in language: Textuality, contextuality, and critique". Applied Linguistics 28/4: 497-517.

Molina, Lucía, Amparo Hurtado Albir (2002). "Translation techniques revisited: A dynamic and functionalist approach". Meta: Translators' Journal 47/4: 498-512.

Mori, Laura (2018). Observing Eurolects: Corpus Analysis of Linguistic Variation in EU Law. Amsterdam - Philadelphia: John Benjamins.

Piller, Ingrid, Jie Zhang, Jia Li (2020). "Linguistic diversity in a time of crisis: Language challenges of the COVID-19 pandemic". Multilingua 39/5: 503-515.

Pimentel, Janine (2017). "Specialized verbs and specialized uses of verbs in a comparable corpus of judgments produced in Canada, Portugal, and Brazil". In: Patrick Drouin, Aline Francœur, John Humbley, Aurélie Picton (eds.). Multiple Perspectives on 
Terminological Variation. Amsterdam - Philadelphia: John Benjamins, 109-130.

Prieto Ramos, Fernando, Albert Morales (2019). "Terminological innovation and harmonization at international organizations: Can too many cooks spoil the broth?". In: Marita Kristiansen, Ingrid Simonnaes (eds.). Legal Translation: Current Issues and Challenges in Research, Methods and Applications. Berlin: Frank \& Timme, 87-110.

Rossi, Micaela (2017). "Terminological metaphors and the nomadism of specialized terms: Some observations on intralinguistic and interlinguistic variation". In: Patrick Drouin, Aline Francœur, John Humbley, Aurélie Picton (eds.). Multiple Perspectives on Terminological Variation. Amsterdam - Philadelphia: John Benjamins, 181212.

Seracini, Francesca L. (2020). "Phraseology in multilingual EU legislation: A corpus-based study of translated multi-word terms". Perspectives. DOI: 10.1080/0907676X.2020.1800058.

Sękowska, Elżbieta (2002). "Neologizmy słowotwórcze we współczesnej polszczyźnie (wybrane tendencje)". Eslavística Complutense 4: 1-9.

Shelov, Sergey D. (2018). Ocherk teorii terminologii: Sostav, ponyatiynaya organizatsiya, prakticheskiye prilozheniya. Moscow: V.V. Vinogradov Russian Language Institute of the Russian Academy of Sciences.

Sosoni, Vilelmini, John O'Shea (2020). "Translating property law terms: an investigation of Greek notarial deeds and their English translations". Perspectives. DOI: 10.1080/0907676X.2020.1797840.

Strouther, Danielle (2020). "The remote working challenges digital businesses face after COVID-19. Search Engine Journal. Available at https://www.searchenginejournal.com/remote-working-challen ges/371448/\#close. Accessed 02.10.2020.

Tomaszewska, Aleksandra, Natalia Zawadzka-Paluektau (2020). "COVID-19 multi-word terminology in EU press releases: Data". RepOD, V1. Available at https://doi.org/10.18150/DU8D8Z. Accessed 01.11.2020.

Weston, Martin (1991). An English Reader's Guide to the French Legal System. New York, Oxford: Berg.

World Health Organization (2020). WHO Coronavirus Disease (COVID19) Pandemic. Available at https://www.who.int/emergencies/diseases/novel-coronavirus-2019. Accessed 26.10.2020.

Zhang, Jie, Yuqin Wu (2020). "Providing multilingual logistics communication in COVID-19 disaster relief". Multilingua 39/5: 517-528. 
Aleksandra Tomaszewska

ORCID iD: 0000-0001-6379-3034

University of Warsaw

Institute of Applied Linguistics

Dobra 55

00-312 Warsaw

Poland

a.tomaszewska2@uw.edu.pl

Natalia Zawadzka-Paluektau

ORCID iD: 0000-0003-4969-2039

University of Warsaw

Institute of English Studies

Hoża 69

00-681 Warsaw

Poland

University of Seville

Department of Spanish Philology

School of Philology

4 San Fernando St.

Seville

Spain

nataliazawadzka@uw.edu.p1 